\title{
Focusing with 2D Square Photonic Crystal with Concavo-Concavo Boundaries
}

\author{
N. Palka*, W. Ciurapinski and J. Wróbel \\ Institute of Optoelectronics, Military University of Technology \\ S. Kaliskiego 2, 00-908 Warsaw, Poland
}

\begin{abstract}
We studied focusing properties of $2 \mathrm{D}$ square photonic crystal with concavo-concavo boundaries. The photonic crystal is built by air holes in a uniform dielectric. The incident plane wave propagates through a section of the photonic crystal and efficient focusing is observed by finite difference time domain simulations. To analyze the properties of photonic crystal we calculated the photonic band structure and equal frequency contours for TE modes. In our simulation we consider influence of geometry of the concave structures on the focusing properties.
\end{abstract}

PACS numbers: $42.70 . \mathrm{Qs}, 42.79 . \mathrm{Bh}, 42.25$.Bs

\section{Introduction}

Photonic crystals (PhCs) are dielectric structures with one-, two- or three-dimensional periodicity. One of their unusual properties is ability to exhibit anomalous refractive effects. Photonic crystals are widely recognized as promising structures for realization of light focusing. Recent research in PhCs offers the possibility of creating a flat "perfect lens" as proposed by Pendry [1]. Such lens can focus a point source and forms a real point image on the other side of the lens. However, this method works only when the source is in the vicinity of the structure. Some groups discovered that far-field focusing can be observed in 2D PhCs with negative refraction and concavo boundaries [2].

Generally, the direction of refracted beams in $\mathrm{PhCs}$ cannot be determined by Snell formula and calculation of the photonic band structure, equal frequency contour (EFC) and some algorithms are required [3].

Our considerations are restricted to terahertz region of wavelength $(0.1-10 \mathrm{~T})$ which seems to be a new and promising area for PhCs.

\section{Refraction in photonic crystal}

In this work we focus only on $2 \mathrm{D} \mathrm{PhCs}$ with square array of air holes in a matrix - silicon, whose refractive index equals to 3.1416 in THz range (Fig. 1a). For the square array the reciprocal zone is also square with dimensions determined in $k$-space. A common characteristic of the $\mathrm{PhC}$ is a band diagram, which depicts wave vectors versus mode frequency of the Bloch waves traveling inside the $\mathrm{PhC}[4]$. The ordinary band diagram

\footnotetext{
* corresponding author; e-mail: npalka@wat.edu.pl
}

shows the states only around the boundaries of the Brillouin zone. In case of refraction, we calculated the band diagram by means of plane wave expansion method taking into account all directions in an irreducible Brillouin zone (Fig. 1b).

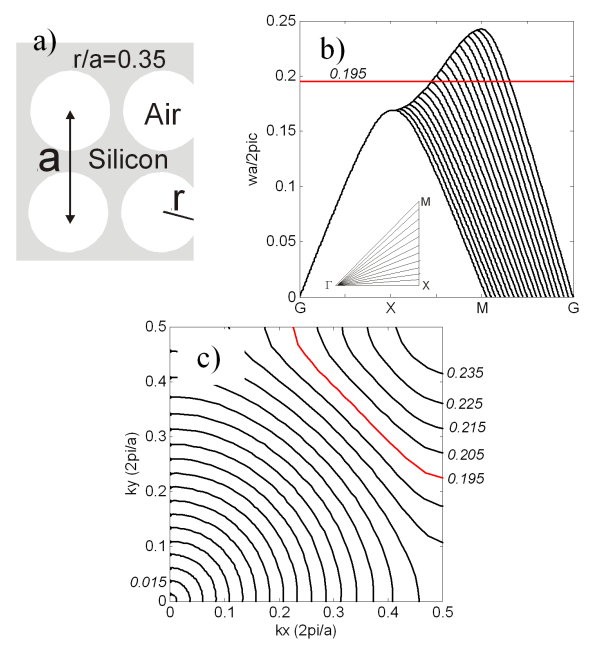

Fig. 1. 2D square photonic crystal (a), the first band of $\mathrm{PhC}$ with all directions in the irreducible Brillouin zone (inset) (b) and equal frequency contour for the first band (c).

When light travels through interference between a homogeneous medium and a $\mathrm{PhC}$ structure, Bloch modes are excited, whose directions can be determined by means of EFC [3-5]. EFC is transformation of coordinates from band diagram (frequency versus wave vector) to isofrequency contours which consist of all allowed propagation modes in wave vector space that exist in the PhC structure for a certain frequency [3]. Direction of the refracted 
wave and its group velocity can be determined from the formula $v_{\mathrm{g}}=\operatorname{grad}_{k} \omega$ thus it is normal to the EFC at a certain wave vector point $k$ and points towards increasing $\omega[3,6]$.

A very interesting feature of the $\mathrm{PhCs}$ is that for nearly flat region of contour, incident beams coming from different directions are nearly parallel inside $\mathrm{PhC}$, because the refracted beams have to be perpendicular to the EFC [4].

\section{Construction of focusing structure}

The PhC-based structure, which exploits the supercollimation effect, has principal optical axis along $\Gamma M$ direction (perpendicular to flat region of EFCs) - Fig. 2. To enhance focusing we removed a set of holes from both sides of the $\mathrm{PhC}$ to create concavo-concavo boundaries with radius $\mathrm{R} 1$ and $\mathrm{R} 2$ [5]. We also used two antireflective layers to improve coupling of light between air/silicon interfaces.

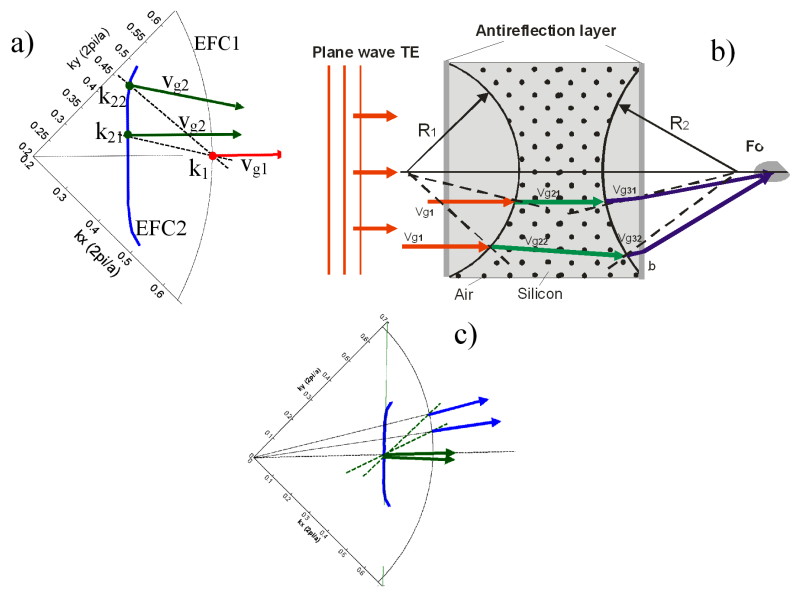

Fig. 2. 2D focusing structure (b), determination of refracted beams: input (a) and output (c) using the EFC-based algorithm.

For $1 \mathrm{THz}$ radiation, silicon refractive index is $n_{\mathrm{Si}}=$ 3.416. The flattest $\mathrm{EFC}$ is for $\omega a / 2 \pi c=0.195$, so the lattice period (a) is equal to $58.5 \mu \mathrm{m}$ and the radius $(r)$ equals to $20.5 \mu \mathrm{m}$ for the assumed ratio $r / a=0.35$.

A plane wave $(1 \mathrm{THz})$ coming from left propagates through the antireflective layer and silicon region and is refracted at the first silicon $/ \mathrm{PhC}$ border. To determine the directions of refracted waves in such structures a following algorithm should be applied - Fig. 2 .

For a chosen frequency, on the isofrequency contour we superimpose EFC1 contour for the incident medium (silicon $-2 \pi n_{\mathrm{Si}} / \lambda$ ) and EFC2 for the transmitted medium $(\mathrm{PhC}) . \mathrm{A}$ dot $k_{1}$ corresponds to an incident wave vector and arrow on the contour - its group velocity $v_{\mathrm{g} 1}$ (a direction of incident beam). Next, we plot a dashed line through the point representing incident wave $k_{1}$. This line is called a construction line - is perpendicular to an interface and represents conservation of parallel part of $k$ vector [4]. The point where the construction line intersects the EFC2 contour determines the refractive wave vector $k_{21}\left(k_{22}\right)$. Direction of refracted wave is normal to the EFC in the point $k_{21}\left(k_{22}\right)$ and points towards increasing $\omega-v_{\mathrm{g} 21}$ and $v_{\mathrm{g} 22}$, respectively. Beams close to center are parallel to optical axis (flat region of EFC2 $k_{21}$ ), while beams further from center are nearly parallel (bent region of $\mathrm{EFC} 2-k_{22}$ ).

Next, these beams travel along the $\mathrm{PhC}$ structure, refract at the second border according to the above algorithm, refract at the silicon/air border and focus.

\section{FDTD simulations}

To validate our consideration we applied 2D finitedifference time-domain (FDTD) method (OptiFDTD by Optiwave), which is a powerful tool in simulation of photonic structures. Structure under consideration is presented in Fig. 3a and has the following geometry: width (along $x$ axis) $-5000 \mu \mathrm{m}(60 \sqrt{2} a)$, thickness (along $z$ axis at $x=0$ ) $-10 \sqrt{2} a \approx 830 \mu \mathrm{m}$, and $R_{1}=R_{2}=3250 \mu \mathrm{m}$. We applied steps equal to $6 \mu \mathrm{m}$ and PML boundaries. Quite efficient focusing of electromagnetic wave is observed (Fig. $3 \mathrm{a}$ and $3 \mathrm{~b}$ ) which agrees well with theoretical considerations.
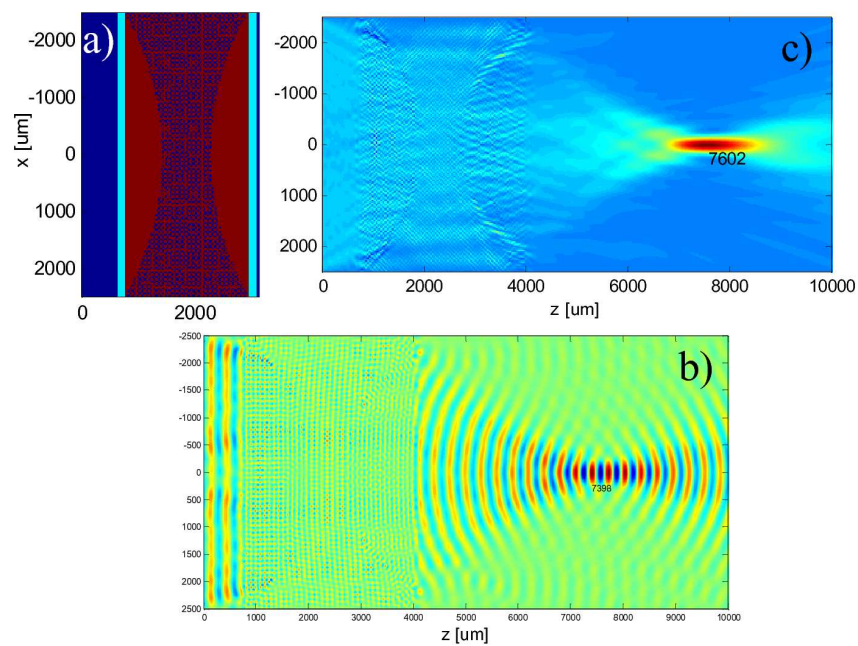

Fig. 3. Simulation of the $\mathrm{PhC}$ structure: layout (a), evolution of electric field - Ey (b) and Poynting vector $-\mathrm{Pz}(\mathrm{c})$.

We studied dependence between geometry of the structure and size of focus. First, we consider influence of radius $\left(R_{1}=R_{2}\right)$ but it was more convenient to use the maximum angle between the edge and the principal axes. Figure 4 presents layouts of the structure and Poynting vectors for angle 30 and 50 degrees. Widths equal to $5000 \mu \mathrm{m}(60 \sqrt{2} a)$, while thicknesses are always equal to $10 \sqrt{2} a$.

Figure 5a presents normalized cross-sections in the focus for angles from 30 to 90 degree. Full width at half 

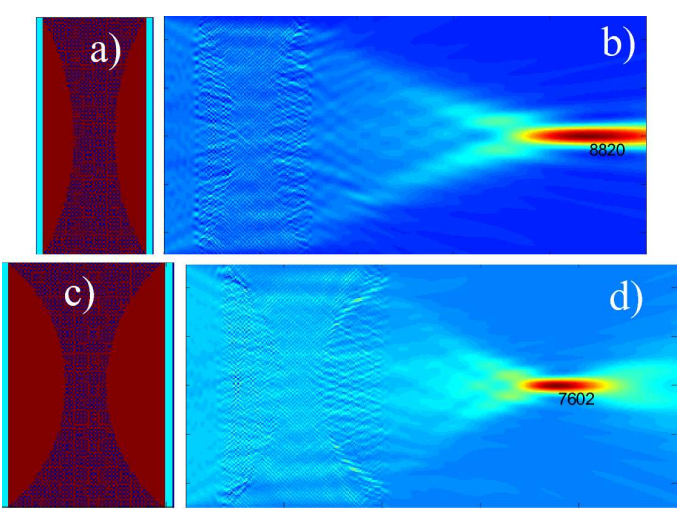

Fig. 4. Layouts of the structure and Poynting vectors for angle 30 (a), (b) and 50 (c), (d) degrees, respectively.

maximum (FWHM) equals to $200 \mu \mathrm{m}$ for 80 and 90 degrees, which is less than wavelength.

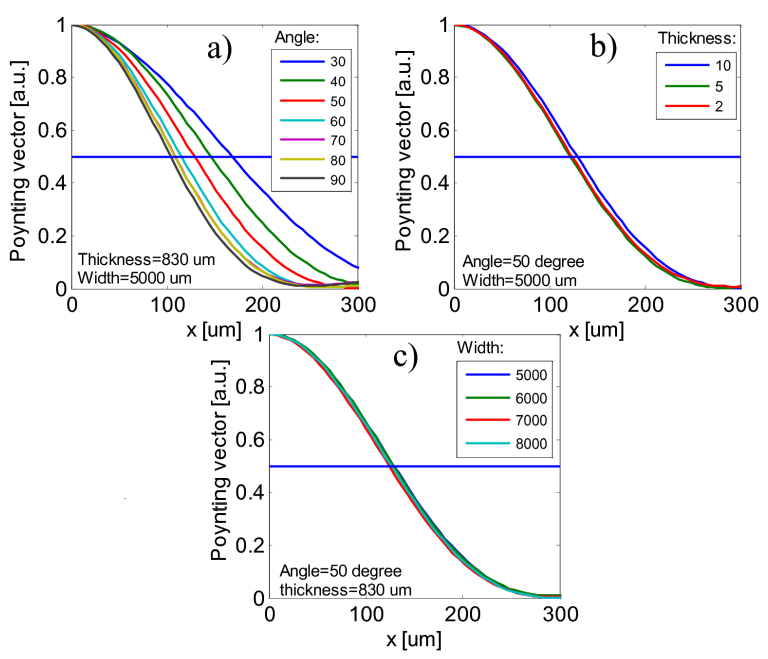

Fig. 5. Normalized cross-sections in focus versus geometry of the structure: angle (a), thickness (b) and width (c).

Next, we examined influence of thickness of the structure (in the narrowest point $x=0$ ) on normalized cross- -section in the focus for 3 values of thickness: $10 \sqrt{2} a \approx$ $830 \mu \mathrm{m}, 5 \sqrt{2} a \approx 415 \mu \mathrm{m}, 2 \sqrt{2} a \approx 165 \mu \mathrm{m}$. Widths equal to $5000 \mu \mathrm{m}(60 \sqrt{2} a)$, while angles are always equal to 50 degree. It is seen that FWHM are nearly the same for these 3 cases (Fig. 5b).

In the end, we focus on dependence between the width of the structure (for 5000, 6000, 7000 and $8000 \mu \mathrm{m}$ ) and cross-section in focus. Thicknesses equal to $830 \mu \mathrm{m}$ $(10 \sqrt{2} a)$, while angles are always equal to 50 degree. Also in this case, FWHM are nearly the same for these 4 cases (Fig. 5c).

\section{Conclusions}

The presented paper deals with refraction phenomenon in the $2 \mathrm{D} \mathrm{PhC}$ and focusing in the concavo-concavo structure. To analyze the properties of $\mathrm{PhC}$ we calculate the photonic band structure and equal frequency contours for TE modes. We examined this structure to be means of fractional differential transform (FDTM) method and obtained quite interesting focusing capabilities. FWHM in focus can be in the range of wavelength.

Moreover, photonic crystals in the $\mathrm{THz}$ range (for $\lambda \approx 300 \mu \mathrm{m}$ crystal lattice $\approx 60 \mu \mathrm{m}$ ) can be easier manufactured in comparison with the visible or infrared structures (for $\lambda \approx 1 \mu \mathrm{m}$ crystal lattice less than $1 \mu \mathrm{m}$ ).

Focusing of good quality is important for novel imaging optics and can lead to considerable changes in optical system design.

\section{References}

[1] J.B. Pendry, Phys. Rev. Lett. 85, 3966 (2000).

[2] Jian Zhi-Jian, Peng Jing-Cui, Zhou Ren-Long, Chinese Phys. Lett. 23, 3359 (2006).

[3] S. Foteinopoulou, C.M. Soukoulis, Phys. Rev. B 72, 9355 (2005).

[4] J.D. Joannopoulos, Photonic Crystal Molding the Flow of Light, Princeton University Press, Manchester 2008, p. 221.

[5] T. Pustelny, M. Grabka, Acta Phys. Pol. A 114, A-113 (2008).

[6] M. Notomi, Phys. Rev. B 62, 10696 (2000). 